\title{
Peripheral T-cell Lymphomas
}

\author{
Dr. Haitham Abdel Bary, MD \\ Lecturer of Internal Medicine, Department of Hematology \\ and Bone Marrow Transplantation, \\ Ain Shams University
}

\section{Introduction}

The peripheral $\mathrm{T}$ cell lymphomas are a heterogeneous group of generally aggressive neoplasms that constitute less than 15 percent of all nonHodgkin lymphomas in adults ${ }^{1}$.

Among these, in decreasing frequency of occurrence, are (Table 1):

-Peripheral T cell lymphoma, not otherwise specified

-Anaplastic large cell lymphoma, primary systemic type

-Angioimmunoblastic T cell lymphoma

-Extranodal NK/T cell lymphoma, nasal type

-Subcutaneous panniculitis-like T cell lymphoma

-Enteropathy associated T cell lymphoma

-Hepatosplenic T cell lymphoma

Peripheral T cell lymphoma, not otherwise specified (PTCL, NOS) is a heterogeneous group of predominantly nodal $\mathrm{T}$ cell lymphomas derived from various types of mature $T$ cells that do not meet the criteria for the other specifically defined subtypes of PTCL listed above. Here in this review we will mainly focus on the clinical picture and management of PTCL,NOS.

Despite their significant differences in pathologic appearance and clinical presentation, the most common entities, peripheral T-cell lymphoma, not otherwise specified (PTCL-NOS), angioimmunoblastic T-cell lymphoma (AITL), and anaplastic lymphoma kinase (ALK)-negative anaplastic large cell lymphoma (ALK-negative ALCL), which account for $; 60 \%$ of cases, tend to be treated similarly². 


\section{EPIDEMIOLOGY}

PTCL, NOS is the most common subtype of PTCL in Western countries, accounting for approximately 30 percent of PTCL and approximately 4 percent of non-Hodgkin lymphomas (NHLs) overall ${ }^{3-4}$.

Most patients are adults with a median age at diagnosis of 60 years 5 . The diagnosis is more common in men than women by a ratio of approximately $2: 1^{6}$.

\section{Table 1. WHO 2008 mature T-cell and NK-cell neoplasms}

Mature T-cell and NK-cell neoplasms Incidence $(\%)^{\star}$

T-cell prolymphocytic leukemia

T-cell large granular lymphocytic leukemia

Chronic lymphoproliferative disorder of NK cells†

Aggressive NK-cell leukemia

Systemic EBV-positive T-cell lymphoproliferative disease of

childhood

Hydroa vacciniforme-like lymphoma

Adult T-cell leukemia/lymphoma 9.6

Extranodal NK/T-cell lymphoma, nasal type

10.4

Enteropathy-associated T-cell lymphoma

Hepatosplenic T-cell lymphoma

Subcutaneous panniculitis-like T-cell lymphoma

0.9

Mycosis fungoides

Sézary syndrome

Primary cutaneous $\mathrm{CD}^{+} 0^{+} \mathrm{T}$-cell lymphoproliferative disorders

Lymphomatoid papulosis

Primary cutaneous anaplastic large cell lymphoma

Primary cutaneous $\gamma \delta \mathrm{T}$-cell lymphoma

Primary cutaneous $\mathrm{CD}^{+}$aggressive epidermotropic cytotoxic

T-cell lymphoma†

Primary cutaneous $\mathrm{CD}^{+}$small/medium T-cell lymphoma†

Peripheral T-cell lymphoma, NOS

Angioimmunoblastic T-cell lymphoma

Anaplastic large cell lymphoma, ALK-positive

Anaplastic large cell lymphoma, ALK-negative†

*Incidence among peripheral T-cell and NK/T-cell lymphoma from the International T-Cell Project. †Provisional entities. 


\section{CLINICAL FEATURES}

Most patients with PTCL, NOS present with generalized lymphadenopathy with or without extranodal disease. Approximately 38 percent of patients have nodal disease alone, 49 percent have nodal and extranodal disease, and 13 percent have extranodal disease without evidence of nodal involvement.

Hepatomegaly and splenomegaly are seen in 17 and 24 percent of patients, respectively. Approximately 14, 17, 26, and 43 percent of cases are stage I, II, III, and IV, respectively. The bone marrow is involved in 20 percent of cases and many others demonstrated infiltration of the liver and/or spleen. While circulating lymphoma cells may be seen, leukemic presentations are rare. Some cases are associated with eosinophilia, pruritus, and/or hemophagocytosis

Thrombocytopenia and anemia are seen in one-quarter of patients ${ }^{6}$.

The skin and gastrointestinal tract are the most commonly involved extranodal $s^{7} e^{7}$. Less commonly involved sites include the lung, salivary gland, and central nervous system. Systemic B symptoms (fever, night sweats, weight loss) are present in approximately 35 percent of patients.

\section{PATHOLOGY}

Histology - Lymph nodes usually demonstrate effacement of the normal architecture by sheets of atypical lymphoid cells in a paracortical or diffuse pattern. The tumor cells have no characteristic morphologic features and typically are comprised of variable mixtures of small, intermediate, and large atypical cells. These cells contain pleomorphic, irregular, vesicular, or hyperchromatic nuclei with prominent nucleoli and usually exhibit a high mitotic rate (Figure 1). They sometimes have clear cytoplasm, a morphologic feature suggestive of a T cell phenotype ${ }^{8}$.

Typically, there are varying numbers of admixed eosinophils, plasma cells, resting or activated $B$ cells, and epithelioid histiocytes (activated macrophages).

Two additional variants include a form characterized by growth in a paracortical pattern, often surrounding reactive B cell follicles, the so-called "T-zone" variant, and another in which the malignant $\mathrm{T}$ cells grow in aggregates within follicles, the so-called "follicular" variant.

Biopsy of involved extranodal tissues demonstrates a diffuse infiltrate of similar atypical lymphoid cells. Skin involvement commonly takes the form of infiltrates in the dermis and subcutis and can result in nodules with central ulceration. Splenic 
involvement can vary from solitary fleshy nodules to diffuse involvement of the white or red pulp 9 .

Figure 1

\section{Peripheral T-cell Iymphoma, not otherwise specified histology}

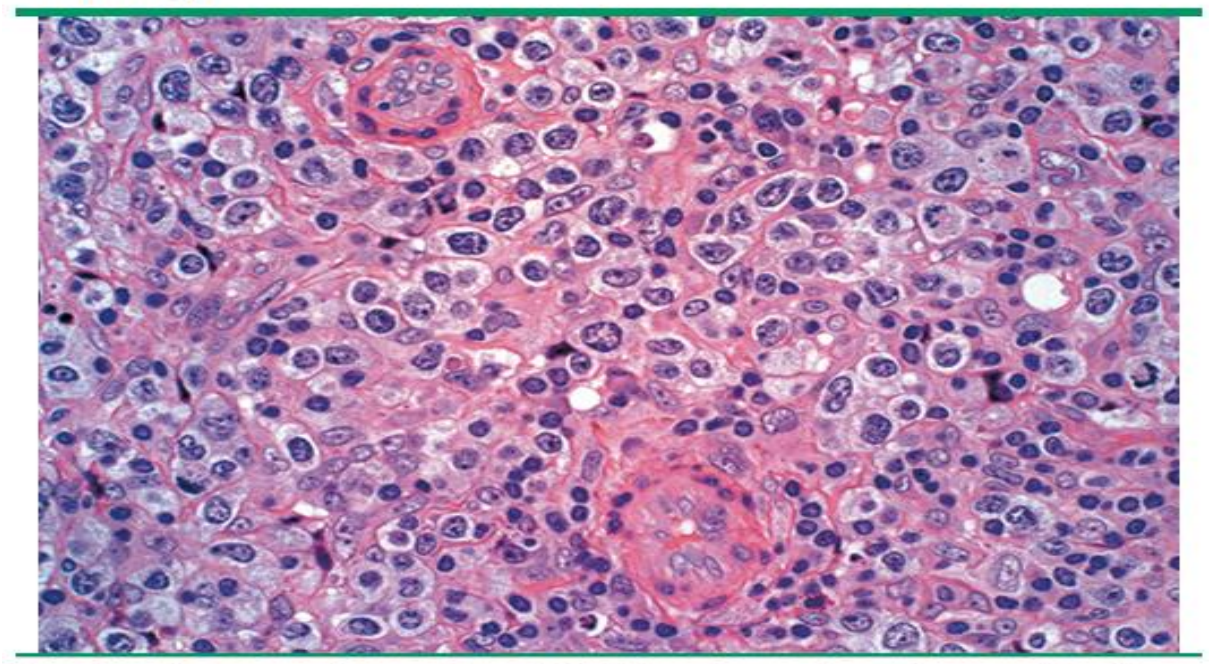

Lymph node biopsy of a peripheral T cell lymphoma shows tumor cells with clear cytoplasm and pleomorphic nuclei. There are two high endothelial venules present in this field. Hematoxylin and eosin, 40x magnification.

\section{Immunophenotype}

There is no characteristic immunophenotype for PTCL, NOS. T cell-associated antigens are variably expressed (CD3+/-, CD2+/-, CD5+/-, CD7-/+). These tumors are generally negative for $B$ cell associated antigens ${ }^{10}$.

In most cases, one or more "mature" T cell antigens are lost, such as CD5 or CD7. Expression of CD4 and CD8 is variable. The majority of tumors express CD4 only, but some tumors may be CD4-/CD8-,CD4-/CD8+, or CD4+/CD8+.

Most cases express alpha/beta T cell receptors (TCR beta positive), while a minority expresses gamma/delta T cell receptors (TCR delta-positive).

\section{Genetic features}

Clonal T cell receptor (TCR) gene rearrangements are usually, but not always, detected; immunoglobulin (Ig) genes are germline. Although approximately 90 
percent of PTCLs demonstrate cytogenetic abnormalities, none is specific for PTCL, NOS. Many patients demonstrate chromosomal gains in 7q, 8q, 17q, and $22 q$, and chromosomal losses in 4q, 5q, 6q, 9p, 10q, 12q, and 13q.

The most common translocations are $t(7 ; 14), t(11 ; 14)$, inv(14), and $t(14 ; 14)$. Analogous to B cell lymphomas where immunoglobulin (lg) loci (BCR genes) are affected, these translocations involve TCR genes located at $14 q 11$ (TCR alpha/delta), 7q34-35 (TCR beta), and 7p15 (TCR gamma).

\section{DIAGNOSIS}

PTCL, NOS is a diagnosis made based upon the results of a tissue biopsy (usually lymph node biopsy) that demonstrates evidence of a $\mathrm{T}$ cell lymphoma that does not meet the criteria for other subtypes of T cell lymphoma.

As described above, histologic examination of the biopsy material usually demonstrates variable mixtures of small, intermediate, and large atypical cells. In many instances, the histologic features are sufficiently atypical (eg, complete nodal effacement by atypical lymphoid cells) that a diagnosis of lymphoma can be made with certainty, but evaluation of the immunophenotype by either immunohistochemistry or flow cytometry is essential to confirm the T cell origin of the tumor. Immunohistochemistry is more reliable, because large lymphoid cells are sometimes lost or disrupted during tissue processing required for performance of flow cytometry.

The immunophenotype of PTCL, NOS varies greatly from case to case, but always demonstrates expression of one or more of the pan-T antigens (ie, CD2, CD3, CD5, CD7). Roughly half of cases demonstrate an aberrant immunophenotype, defined by the loss of a pan-T cell antigen that is expressed by most normal mature $\mathrm{T}$ cells (eg, CD5 or CD7). Detection of surface CD3 (best assessed by flow cytometry) can also be helpful, since this finding excludes rare lymphomas of natural killer cell origin. In difficult or equivocal cases, molecular detection of a clonal T cell receptor (TCR) rearrangement, performed using a PCR-based assay, can be very helpful. While the majority of PTCL, NOS has cytogenetic abnormalities, none are specific.

DIFFERENTIAL DIAGNOSIS - The differential diagnosis of PTCL, NOS includes other T cell lymphomas and B cell lymphomas (including Hodgkin lymphoma) that have similar morphologic and/or immunophenotypic features, and benign disorders that result in hyperplasia of nodal paracortical $\mathrm{T}$ cell zones, such as 
hypersensitivity reactions and unusually prolonged or robust immune responses to viral infections (eg, with Epstein Barr virus).

\section{-Angioimmunoblastic T cell lymphoma}

Angioimmunoblastic T cell lymphoma (AITL) is another relatively common subtype of $\mathrm{T}$ cell lymphoma that presents with generalized lymphadenopathy and systemic symptoms. Like PTCL, NOS, involved lymph nodes demonstrate partial or complete effacement of the normal architecture by a predominantly paracortical infiltrate of atypical lymphoid cells of different sizes and inflammatory cells. There is prominent neovascularization with arborizing high endothelial venules and an expansion of follicular dendritic cells that often surround tumor cells with abundant clear cytoplasm.

The distinction between PTCL, NOS and AITL is based upon the absence of morphologic features typical of AITL and the immunophenotype of the tumor cells. Unlike AITL, the tumor cells in PTCL, NOS do not typically express CD10, BCL6, PD1, or CXCL13. ${ }^{\text {. }}$

\section{- Anaplastic large cell lymphoma}

Anaplastic large cell lymphoma (ALCL) is a lymphoma of $T$ cell or null cell type that typically presents with painless lymphadenopathy with or without systemic symptoms. On histology, the tumor is composed in part of large lymphoma cells with horseshoe-shaped nuclei and prominent nucleoli (hallmark cells). ALCL demonstrates homogeneous, strong expression of CD30 in a membrane and golgi pattern.

ALCL is often positive for epithelial membrane antigen and ALK, the presence of ALK gene rearrangements is due to translocation between chromosomes 2 and 5 , resulting in a fusion protein that joins the $\mathrm{N}$-terminus of nucleophosmin (NPM) to the C-terminus of anaplastic lymphoma kinase (ALK). all of which are absent from PTCL, NOS.

It is important to note that anaplastic large cell lymphoma that is ALK-negative is not always easily distinguished from CD30+ PTCL, NOS, and yet is considered a distinct entity in the 2016 revision of the WHO classification scheme.

-Adult T cell leukemia-lymphoma - Adult T cell leukemia-lymphoma (ATL) is another $\mathrm{T}$ cell lymphoma that typically presents with generalized 
lymphadenopathy, hepatosplenomegaly, immunosuppression, hypercalcemia, lytic bone lesions, and/or skin lesions. The malignant cell of origin in ATL is a transformed HTLV-I infected mature helper (CD4+) T-lymphocyte. Nodal ATL cannot be reliably distinguished from PTCL, NOS on morphologic or immunophenotypic grounds and can only be excluded with certainty if HTLV-I studies (generally serology) are negative, particularly in patients from regions where HTLV-1 is endemic 6 .

-Hodgkin lymphoma - Both PTCL, NOS and classical Hodgkin lymphoma (HL) may contain cells resembling Reed-Sternberg cells in an inflammatory background. Uncommonly, PTCL, NOS can express CD30 and rarely are positive for CD15, markers that are typically positive in classical forms of HL. However, PTCL, NOS is differentiated from HL by the absence of PAX5, a B cell-specific factor seen in most cases of $\mathrm{HL}$, and by the expression of alpha/beta $\mathrm{T}$ cell receptors (TCR beta positive) and other T cell markers.

\section{OVERVIEW OF TREATMENT}

Despite their significant differences in pathologic appearance and clinical presentation, the most common entities, peripheral T-cell lymphoma, not otherwise specified (PTCL-NOS), angioimmunoblastic T-cell lymphoma (AITL), and anaplastic lymphoma kinase (ALK)-negative anaplastic large cell lymphoma (ALKnegative $A L C L)$, which account for $; 60 \%$ of cases, tend to be treated similarly.

\section{Outcomes with standard therapy: cyclophosphamide, doxorubicin,} vincristine, and prednisone:

The ITCP and BCCA series are useful in informing us on the expected survival outcomes with $\mathrm{CHOP}$ (cyclophosphamide, doxorubicin, vincristine, prednisone) in PTCL. In the ITCP, $85 \%$ of patients received CHOP-based therapy, and in contrast to ALK positive ALCL where the 5-year failure-free survival (FFS) was $60 \%$, the 5 year FFS for PTCL-NOS, AITL, and ALK-negative ALCL were only $20 \%, 18 \%$, and $36 \%$, respectively. Similar outcomes were observed in the BCCA series with 5year progression-free survival (PFS) of $29 \%, 13 \%$, and $28 \%$ for PTCL-NOS, AITL, and ALCL, respectivel ${ }^{12}$.

A randomized phase III trial comparing CHOP chemotherapy with a more intensive chemotherapy regimen (etoposide, ifosfamide, cisplatin alternating with doxorubicin,bleomycin,vinblastine and dacarbazine) in 88 patients with newly 
diagnosed PTCL reported no difference in event-free survival at two years, but greater toxicity with the more intensive regimen ${ }^{13}$.

In addition, a single institution retrospective analysis of 135 patients with PTCL reported no improvement in three-year overall survival rates when $\mathrm{CHOP}$ was compared with more intensive regimens such as HyperCVAD ${ }^{14}$.

While highly intensive chemotherapy regimens do not appear to improve outcomes, data suggest that adding etoposide to standard dose $\mathrm{CHOP}$ for younger patients with PTCL improves event-free survival but does not improve overall survival, A post-hoc analysis evaluated outcomes from 320 patients with PTCL treated on prospective trials with six to eight cycles of $\mathrm{CHOP}$ or $\mathrm{CHOP}$ plus etoposide (CHOEP) and followed for a median of 44 months, For patients younger than age 60 who had a normal LDH at diagnosis, CHOEP resulted in a significantly higher rate of event-free survival at three years when compared with CHOP (75 versus 51 percent, respectively).

The consensus suggests induction chemotherapy with a CHOP-based regimen rather than more intensive induction regimens such as $\mathrm{EPOCH}$ or HyperCVAD. For patients younger than 60 years, The suggestion is to use of CHOEP rather than CHOP. This preference places a high importance on the improved event-free survival seen with CHOEP with the understanding that adding etoposide will increase toxicity. Patients over the age of 60 years are generally treated with CHOP in order to avoid the increased toxicity.

\section{CONSOLIDATION THERAPY}

The role of consolidation therapy in PTCL is controversial. However, there is a general consensus that HCT is not indicated after the attainment of a first complete remission in the following two subgroups:

- Most patients with ALK positive anaplastic large cell lymphoma (ALCL). Such patients have five-year overall survival rates ranging from 70 to 93 percent after anthracycline-based chemotherapy.

-Patients with localized PTCL, not otherwise specified (PTCL, NOS) and a low or low intermediate International Prognostic Index (IPI) score. Five-year overall survival rates are 74 and 49 percent among patients with a low (ie, zero to 1) or low intermediate (ie, 2) IPI score, respectively. 
PTCL patients who do not fit into these two categories have five-year overall survival rates of 20 percent or less after combination chemotherapy alone. Such patients should be considered for consolidation therapy.

\section{Transplantation}

\section{Autologous}

The largest study was the Nordic study by d'Amore and colleagues. This study enrolled 160 patients with PTCL, including 39\% with PTCL-NOS, 19\% with ALKnegative ALCL, and 19\% with AITL, and excluded ALK-positive ALCL.

Most patients (81\%) presented with advanced stage disease and $72 \%$ had IPI scores of 2 or more. Patients received CHOEP for 6 cycles (etoposide was omitted for patients .60 years of age) and those in CR or partial response proceeded to high-dose therapy with carmustine, etoposide, cytarabine, and melphalan (or cyclophosphamide) and ASCT. One hundred fifteen (71\%) patients underwent ASCT.

By intent-to-treat analysis, the 5-year OS and PFS were $51 \%$ and $44 \%$, respectively. The patients with ALK-negative ALCL performed particularly well, with 5-year OS and PFS of $70 \%$ and $61 \%$, respectively. The 5-year OS and PFS for patients with PTCL,NOS were $47 \%$ and $38 \%$, respectively, and for AITL were $52 \%$ and $49 \%$, respectively ${ }^{16}$.

Reimer and colleagues conducted the second largest prospective study evaluating ASCT in first remission after CHOP, which enrolled 83 patients, By intent-to-treat analysis, similar results were seen, with a 3 -year OS rate at $48 \%$. For those who were transplanted ( $66 \%$ of patients enrolled), outcomes were considerably more favorable, with a 3 -year OS of $71 \%{ }^{17}$.

Radiation - With the exception of extranodal NK/T cell lymphoma, the role of radiotherapy $(R T)$ in managing $P T C L$ remains undefined. In general, radiotherapy is offerd in the following situations:

-Patients with localized (stage I or II) disease who achieve a complete remission (CR) with chemotherapy.

-Some patients with localized disease who achieve a partial remission with chemotherapy. RT is generally offered to such patients who are not transplant (HCT) candidates. 


\section{MODIFICATION FOR SUBTYPES}

Although the most common peripheral T cell lymphoma (PTCL) histology is PTCL, not otherwise specified (PTCL, NOS), there are several subtypes of PTCL with unique features that require treatment modifications:

Anaplastic large cell lymphoma - Anaplastic large cell lymphoma (ALCL) has a more favorable outcome than other subtypes of PTCL, particularly in those cases expressing the ALK protein. In general, patients are treated with the same induction therapy regimens used for PTCL, NOS. Postremission therapy is dependent upon the patient's age and comorbidities along with the ALK status of the tumor.

Angioimmunoblastic $\mathbf{T}$ cell lymphoma - There is no consensus regarding the preferred initial treatment of angioimmunoblastic $T$ cell lymphoma (AITL). Treatment options include steroids, combination chemotherapy, auto-HCT, and investigational therapies. Although complete remission $(\mathrm{CR})$ can be attained in many patients, relapse is frequent, with median overall survival (OS) rates ranging from 15 to 36 months with chemotherapy and of approximately four years with auto-HCT.

Hepatosplenic T cell lymphoma - There are limited data on the treatment of hepatosplenic $\mathrm{T}$ cell lymphoma. While up to half of patients may achieve a complete remission (CR) with chemotherapy, remissions are typically short lived with a median overall survival of approximately one year. No standard therapy exists, though autologous or even allogeneic transplantation should be considered in patients who achieve a CR. Although the data to support these aggressive strategies are limited, the outcome with chemotherapy alone is poor.

Extranodal NK/T cell lymphoma, nasal type - The treatment of patients with extranodal NK/T cell lymphoma, nasal type is largely determined by the extent of disease. Localized disease is commonly treated with radiation therapy with or without chemotherapy while patients with advanced disease are treated with combination chemotherapy with or without hematopoietic cell transplantation ${ }^{18}$. 


\section{TREATMENT OF RELAPSED/REFRACTORY DISEASE}

\section{Chemotherapy}

Traditional regimens - Patients who are transplant candidates may respond to traditional regimens used for aggressive lymphoma such as:

-ICE (ifosfamide, ncarboplatin, etoposide) ${ }^{19}$.

-DHAP (edexamethasone, high dose cytarabine, and cisplatin) ${ }^{20}$.

-ESHAP (etoposide, methyl-prednisolone, cytarabine, and cisplatin) ${ }^{21}$.

-Single agent gemcitabine ${ }^{22}$..

-GDP (Gemcitabine, cisplatin, and dexamethasone) $)^{23}$.

- Gemcitabine and oxaliplatin ${ }^{24}$.

\section{Novel agents}

Pralatrexate - Initial trials of the novel antifolate Pralatrexate have demonstrated encouraging response rates in PTCL:

An international, open label phase II registration trial (the PROPEL trial) evaluated the use of Pralatrexate in 115 heavily pretreated patients with PTCL. Patients received pralatrexate $30 \mathrm{mg} / \mathrm{m}^{2}$ weekly for six of every seven weeks along with folic acid $1 \mathrm{mg}$ by mouth daily and vitamin B12 injections monthly. Patients were heavily pretreated with a median of three prior therapies (range 1 to 12) and 16 percent of patients had failed a prior autologous HCT, The overall response rate (ORR) was 29 percent with 18 percent partial responses (PR) and 11 percent complete response (CR) or CR, unconfirmed. Twenty-one patients (19 percent) had stable disease. The median time on therapy for all responders was 186 days and the median duration of response was 10 months. The median overall and progression free survival times were 14.5 and 3.5 months, respectively.

Pralatrexate is approved for use in relapsed or refractory PTCL by the United States Food and Drug Administration ${ }^{25}$.

\section{Romidepsin}

Romidepsin is a histone deacetylase inhibitor that has demonstrated responses in relapsed or refractory PTCL in phase II trials, Romidepsin was granted 
accelerated approval by the US Food and Drug Administration for the treatment of patients with PTCL who have received at least one prior therapy.

A phase II trial of romidepsin (14 mg/m² on days 1,8 , and 15 of a 28-day cycle) in 45 patients with relapsed or refractory PTCL who had received a median of three prior therapies showed an overall response rate (ORR) of 38 percent (eight complete, nine partial). The median duration of response was nine months (range 2 to 74 months) ${ }^{26}$.

An international phase II trial of romidepsin $\left(14 \mathrm{mg} / \mathrm{m}^{2}\right.$ on days 1,8 , and 15 of a 28-day cycle) in 130 patients with relapsed or refractory PTCL who had received a median of two prior therapies showed an ORR of 25 percent (15 percent complete). Responses were similar in all subtypes of PTCL. Objective responses were seen at a median of two months and complete responses were seen at a median of 3.7 months $^{27}$.

\section{Belinostat}

Belinostatis a histone deacetylase inhibitor that has demonstrated responses in relapsed or refractory PTCL in a phase II trial. Belinostat was granted accelerated approval by the US Food and Drug Administration for the treatment of patients with PTCL who have received at least one prior therapy.

A phase II trial (BELIEF trial) of belinostat $\left(1000 \mathrm{mg} / \mathrm{m}^{2}\right.$ days 1 to 5 of a 21 -day cycle) in 120 evaluable patients with PTCL reported overall and complete remission rates of 26 and 11 percent, respectively, with a median time to response of 5.6 weeks (range 4 to 50 weeks). The median duration of response was 14 months (>29 months among those achieving CR) ${ }^{28}$.

\section{Brentuximab}

Brentuximab vedotin (commonly referred to as brentuximab) is an antibody drug conjugate with a CD-30 directed antibody linked to the antitubulin agent monomethyl auristatin E (MMAE). Brentuximab is approved by the US Food and Drug Administration for the treatment of patients with systemic ALCL after failure of at least one prior multi-agent chemotherapy regimen.

Brentuximab has also demonstrated activity in other histologic subtypes of CD30positive PTCL, but is not approved by regulatory agencies for this indication. In a single arm, phase II, multicenter trial, 35 patients with relapsed/refractory CD30- 
positive PTCL received single agent brentuximab $(1.8 \mathrm{mg} / \mathrm{kg}$ every three weeks until disease progression or unacceptable toxicity). The overall response rate was 41 percent (24 percent complete). Among the 13 patients with angioimmunoblastic T cell lymphoma, five attained a complete remission (CR) and two had a partial remission (PR), with a median progression-free survival of 6.7 months. Among the 21 patients with PTCL, NOS, three attained a CR and four had a PR, with a median progression-free survival of 1.6 month $^{29}$.

\section{Transplantation}

Allogeneic HCT - Allogeneic hematopoietic cell transplantation (allo-HCT) has been studied in a selective group of patients with relapsed or refractory PTCL. Rates of overall survival at five years post-transplant are approximately 50 to 60 percent, with non-relapse mortality (NRM) rates of 20 to 25 percent.

Analysis of the Center for International Blood and Marrow Transplantation Research (CIBMTR) database identified 112 patients with PTCL who had undergone allo-HCT in first complete remission (CR, 14 percent), second or greater CR (16 percent), primary induction failure (36 percent), chemotherapy sensitive relapse (17 percent), or other stage of disease.

The estimated rates of progression-free survival and overall survival at one year were 42 and 55 percent, respectively. Corresponding rates at three years were 37 and 46 percent. Non-relapse mortality was 34 percent at three years. Survival was worse for patients not in $\mathrm{CR}$ at the time of HCT and for those who had received more than two lines of chemotherapy ${ }^{15,30}$.

\section{Autologous HCT}

When compared with patients transplanted in first complete remission (CR), overall and progression free survival rates are significantly lower when auto-HCT is performed on patients in subsequent $C R$, partial remission (PR), or those with progressive disease.

An analysis of the Center for International Blood and Marrow Transplantation Research (CIBMTR) database identified 115 patients with PTCL (including ALCL) undergoing auto-HCT in first CR (35 percent), second or greater CR (21 percent), primary induction failure (19 percent), chemotherapy sensitive relapse (15 percent), or other (11 percent). The estimated rates of progression-free survival and overall survival at one year were 58 and 68 percent, respectively. 
Corresponding rates at three years were 47 and 59 percent. Non-relapse mortality at one and three years was 4 and 6 percent ${ }^{15,30}$.

\section{Bortezomib}

A phase II trial of bortezomib (1.3 mg/m² on days $1,4,8$, and 11 of a 21 -day cycle) in 15 patients with relapsed/refractory PTCL (two patients) or cutaneous $\mathrm{T}$ cell lymphoma (CTCL) reported an overall response rate (ORR) of 67 percent with two complete $(\mathrm{CR})$ and six partial remissions. Of the two patients with PTCL, one attained a CR. The responses were durable, lasting on average 7 to 14 months ${ }^{31}$.

\section{Bendamustine}

An open-label, phase 2 trial evaluated the use of bendamustine in 60 patients with relapsed or refractory PTCL. The overall response rate was 50 percent (28 percent complete). The median duration of response, progression-free survival, and overall survival were $3.5,3.6$, and 6.2 months, respectively ${ }^{32}$.

Lenalidomide - A phase 2 trial evaluated the use of lenalidomide ( $25 \mathrm{mg}$ daily on days 1 to 21 of a 28 -day cycle) in 31 patients with relapsed or refractory $T$ cell lymphoma and 8 patients with previously untreated $T$ cell lymphoma who were not candidates for combination chemotherapy. Ten patients had responsive disease (three complete, seven partial), and three patients had stable disease for at least five cycles. The estimated median overall survival and progression-free survival were 12 and 4 months, respectively. The median duration of response was 13 months ${ }^{33}$.

\section{References}

1. Swerdlow SH, Campo E, Harris NL, et al. (Eds). World Health Organization Classification of Tumours of Haematopoietic and Lymphoid Tissues, IARC Press, Lyon 2008.

2. Moskowitz AJ, Lunning MA, Horwitz SM. How I treat peripheral T-cell lymphomas. Blood 2014;17:2636-2644.

3. Abramson JS,Feldman T,Kroll-Desrosiers AR et al. Peripheral T-cell lymphomas in a large US multicenter cohort:prognosticationin the modern era including impact of front line therapy. Ann Oncol 2014;25:2211. 
4.Ellin F,Landström J,Jerkeman M,Relander T. Real-world data on prognostic factors and treatment in peripheral T-cell lymphoma:a study from the Swedish lymphoma registry. Blood. 2014;124:1570.

5.Vose J, Armitage J, Weisenburger D, International T-Cell Lymphoma Project. International peripheral T-cell and natural killer/T-cell lymphoma study: pathology findings and clinical outcomes. J Clin Oncol 2008:26:4124.

6.Weisenburger DD, Savage KJ, Harris NL, et al. Peripheral T-cell lymphoma, not otherwise specified: a report of 340 cases from the International Peripheral Tcell Lymphoma Project. Blood 2011; 117:3402.

7. Bekkenk MW, Vermeer MH, Jansen PM, et al. Peripheral T-cell lymphomas unspecified presenting in the skin: analysis of prognostic factors in a group of 82 patients. Blood 2003; 102:2213.

8.Suchi T, Lennert K, Tu LY, et al. Histopathology and immunohistochemistry of peripheral T cell lymphomas: a proposal for their classification. J Clin Pathol 1987; 40:995.

9. Harris NL, Jaffe ES, Stein $\mathrm{H}$, et al. A revised European-American classification of lymphoid neoplasms: a proposal from the International Lymphoma Study Group. Blood 1994; 84:1361..

10. Borowitz MJ, Reichert TA, Brynes RK, et al. The phenotypic diversity of peripheral T-cell lymphomas: the Southeastern Cancer Study Group experience. Hum Pathol 1986; 17:567.

11.Ong ST, Le Beau MM. Chromosomal abnormalities and molecular genetics of non-Hodgkin's lymphoma. Semin Oncol 1998; 25:447.

12. Savage KJ, Chhanabhai M, Gascoyne RD, Connors JM. Characterization of peripheral T-cell lymphomas in a single North American institution by the WHO classification. Ann Oncol. 2004; 15(10):1467-1475.

13. Simon A, Peoch M, Casassus $P$, et al. Upfront VIP-reinforced-ABVD (VIPrABVD) is not superior to $\mathrm{CHOP} / 21$ in newly diagnosed peripheral $\mathrm{T}$ cell lymphoma. Results of the randomized phase III trial GOELAMS-LTP95. Br J Haematol 2010; 151:159.

14. Escalón MP, Liu NS, Yang Y, et al. Prognostic factors and treatment of patients with T-cell non-Hodgkin lymphoma: the M. D. Anderson Cancer Center experience. Cancer 2005; 103:2091.

15. Smith SM, Burns LJ, van Besien K, et al. Hematopoietic cell transplantation for systemic mature T-cell non-Hodgkin lymphoma. J Clin Oncol 2013; 31:3100. 
16. d'Amore F, Relander T, Lauritzsen GF, et al. Up-front autologous stem-cell transplantation in peripheral T-cell lymphoma: NLG-T-01. J Clin Oncol.

2012;30(25):3093-3099

\section{Reimer P, Rudiger T, Geissinger E, et al. Autologous stem-cell}

transplantation as first-line therapy in peripheral T-cell lymphomas: results of a prospective multicenter study. J Clin Oncol. 2009;27(1):106-113.

18. Falchook GS, Vega F, Dang NH, et al. Hepatosplenic gamma-delta T-cell lymphoma: clinicopathological features and treatment. Ann Oncol 2009; 20:1080.

19. Zelenetz AD, Hamlin P, Kewalramani T, et al. Ifosfamide, carboplatin, etoposide (ICE)-based second-line chemotherapy for the management of relapsed and refractory aggressive non-Hodgkin's lymphoma. Ann Oncol 2003; 14 Suppl 1:i5.

20.Velasquez WS, Cabanillas F, Salvador P, et al. Effective salvage therapy for lymphoma with cisplatin in combination with high-dose Ara-C and dexamethasone (DHAP). Blood 1988; 71:117.

21.Velasquez WS, McLaughlin P, Tucker S, et al. ESHAP--an effective chemotherapy regimen in refractory and relapsing lymphoma: a 4-year follow-up study. J Clin Oncol 1994; 12:1169.

22.Zinzani PL, Venturini F, Stefoni V, et al. Gemcitabine as single agent in pretreated T-cell lymphoma patients: evaluation of the long-term outcome. Ann Oncol 2010; 21:860.

23.Crump M, Baetz T, Couban S, et al. Gemcitabine, dexamethasone, and cisplatin in patients with recurrent or refractory aggressive histology B-cell nonHodgkin lymphoma: a Phase II study by the National Cancer Institute of Canada Clinical Trials Group (NCIC-CTG). Cancer 2004; 101:1835.

24.López A, Gutiérrez A, Palacios A, et al. GEMOX-R regimen is a highly effective salvage regimen in patients with refractory/relapsing diffuse large-cell lymphoma: a phase II study. Eur J Haematol 2008; 80:127.

25. O'Connor OA, Pro B, Pinter-Brown L, et al. Pralatrexate in patients with relapsed or refractory peripheral T-cell lymphoma: results from the pivotal PROPEL study. J Clin Oncol 2011; 29:1182.

26. Piekarz RL, Frye R, Prince HM, et al. Phase 2 trial of romidepsin in patients with peripheral T-cell lymphoma. Blood 2011; 117:5827. 
27.Coiffier B, Pro B, Prince HM, et al. Results from a pivotal, open-label, phase II study of romidepsin in relapsed or refractory peripheral T-cell lymphoma after prior systemic therapy. J Clin Oncol 2012; 30:631.

28. O'Connor OA, Horwitz S, Masszi T, et al. Belinostat in Patients With Relapsed or Refractory Peripheral T-Cell Lymphoma: Results of the Pivotal Phase II BELIEF (CLN-19) Study. J Clin Oncol 2015; 33:249.

29. Horwitz SM, Advani RH, Bartlett NL, et al. Objective responses in relapsed Tcell lymphomas with single-agent brentuximab vedotin. Blood 2014; 123:3095.

30. Smith SM, Burns LJ, van Besien K, et al. Hematopoietic cell transplantation for systemic mature T-cell non-Hodgkin lymphoma. J Clin Oncol 2013; 31:3100.

31. Zinzani PL, Musuraca G, Tani M, et al. Phase II trial of proteasome inhibitor bortezomib in patients with relapsed or refractory cutaneous T-cell lymphoma. J Clin Oncol 2007; 25:4293.

32. Damaj G, Gressin R, Bouabdallah K, et al. Results from a prospective, openlabel, phase II trial of bendamustine in refractory or relapsed T-cell lymphomas: the BENTLY trial. J Clin Oncol 2013; 31:104.

33. Toumishey E, Prasad A, Dueck G, et al. Final report of a phase 2 clinical trial of lenalidomide monotherapy for patients with T-cell lymphoma. Cancer 2015; $121: 716$.

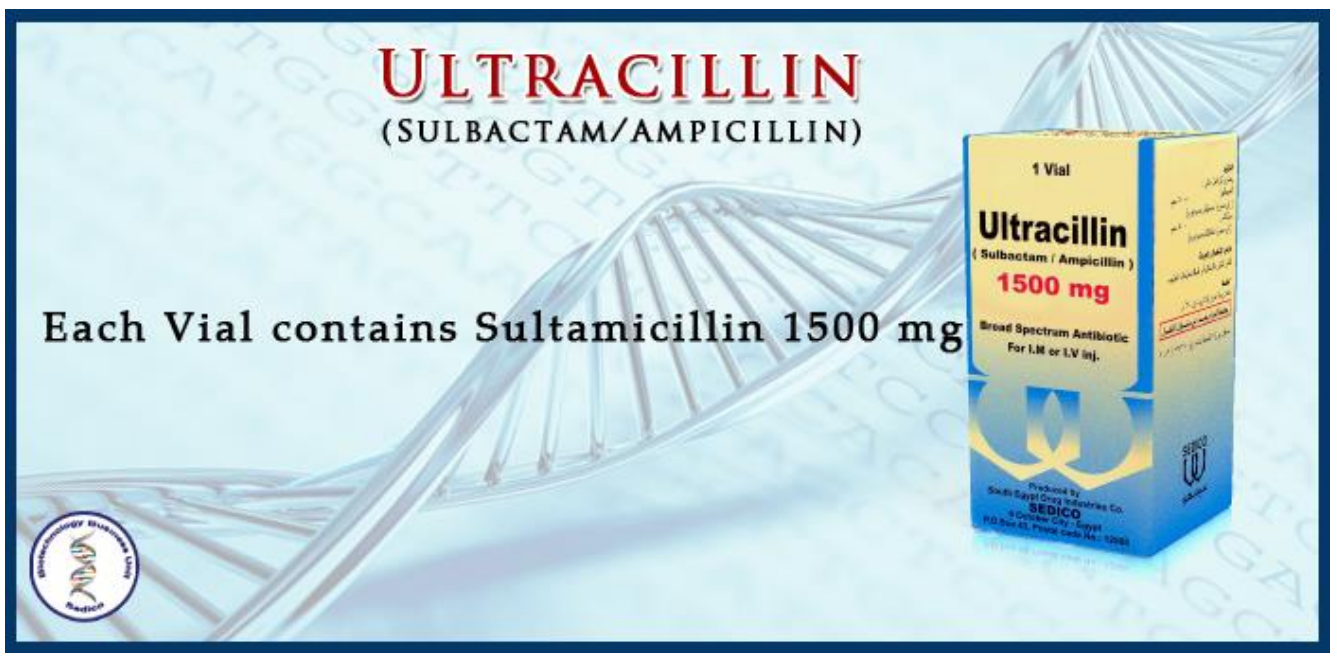

